\title{
Q Fever in Small Ruminants and its Public Health Importance
}

\author{
Tolera Tagesu* \\ School of Veterinary Medicine, Jimma University, Ethiopia
}

Submission: December 01, 2018; Published: January 11, 2019

*Corresponding author: Tolera Tagesu Tucho, School of Veterinary Medicine, Jimma University, Jimma Oromia, Ethiopia

\begin{abstract}
Query fever is caused by Coxiella burnetii, it's a worldwide zoonotic infectious disease where domestic small ruminants are the main reservoirs for human infections. Coxiella burnetii, is a Gram-negative obligate intracellular bacterium, adapted to thrive within the phagolysosome of the phagocyte. Humans become infected primarily by inhaling aerosols that are contaminated with $C$. burnetii. Ingestion (particularly drinking raw milk) and person-to-person transmission are minor routes. Animals shed the bacterium in urine and feces, and in very high concentrations in birth by-products. The bacterium persists in the environment in a resistant spore-like form which may become airborne and transported long distances by the wind. It is considered primarily as occupational disease of workers in close contact with farm animals or processing their products, however, it may occur also in persons without direct contact. Doxycycline drug is the first line of treatment for $Q$ fever. Treatment should be commenced immediately whenever $\mathrm{Q}$ fever is suspected. To prevent both the introduction and spread of $\mathrm{Q}$ fever infection, preventive measures should be implemented including immunization with currently available vaccines of domestic small ruminant animals and humans at risk. And controlling environmental contamination through the control of infected ticks and biosecurity measures may reduce introduction of $C$. burnetii to the farms. To sum up, the objective of this seminar paper is to give highlight on various aspects of $Q$ fever in small ruminants with emphasis on its epidemiology, diagnosis, and its public health importance.
\end{abstract}

Keywords: Coxiella burnetiid; Goats; Q fever; Sheep; Zoonosis

Abbreviations: ACCM: Acidified Citrate Cysteine Medium; LPS: Lipopolysaccharide; RFLP: Restriction Fragment Length Polymorphism; LCV: Large Cell Variant; SCV: Spore-Like Small Cell Variant; CFT: Compliment Fixation Test; IFA: Immunofluorescence Assay; ELISA: Enzyme Linked Immunosorbent Assays; BSL: Bio-Safety Level; MLVA: Multi-Locus Variable number of tandem repeats Analysis; MST: Multispacer Sequence Typing

\section{Introduction}

$\mathrm{Q}$ fever is a zoonotic disease caused by a unique intracellular bacterium Coxiella burnetii, which is a small obligate intracellular gram-negative bacterium that is prevalent throughout the world except in New Zealand and Antarctica [1]. Recently, this intracellular bacterium was classified into the Legionellales order and the Coxiellaceae family [2]. Infection with $C$. burnetii has been detected in humans and a wide range of animal species [3]. The economic and public health impacts of $Q$ fever remain a major concern in developing countries because $Q$ fever causes significant loss of animal productivity and is a zoonotic risk to humans [4]. Human infection, through the inhalation of contaminated aerosols, can cause a broad spectrum of presentations ranging from asymptomatic infection to life-threatening systemic infection. However, the consumption of milk and dairy products, skin contact, and person to person transmission is other routes of transmission of the infection [5]. A small proportion of Coxiella burnetii infections are chronic and predominantly manifested as 'endocarditis' years after exposure. Interestingly, a recent study confirmed that, even with adequate antibiotic treatment, $Q$ fever has a significant long-term health impact with more than one in three patients continuing to suffer from an impaired health status 24 months post-diagnosis [6].

Small ruminants are the primary reservoir for $C$. burnetii infection. Infection from these, predominantly dairy goats, and sheep, is generally subclinical. Although Coxiella can trigger abortion, the placenta and birthing fluid contains a substantial bacterial load that contaminates the local environment [7]. Such contamination is a significant threat to both the agricultural industry and public health. This was recently demonstrated in the Netherlands where widespread Coxiella infection of goats, in a region of substantial goat farming, led to the largest $Q$ fever outbreak ever recorded [8]. During the period of 2007 and 2011, over 4000 human cases of Q fever were diagnosed in the Netherlands [9]. The morbidity (the sickness) and mortality (the death) due to $Q$ fever, in combination with the extremely low infectious dose and environmental stability of the agent, gave rise to the US Centers for Disease Control and Prevention classifying Coxiella burnetii as a biological weapon agent [10]. Up to date, researchers are trying to investigate the molecular mechanisms of Coxiella burnetii pathogenesis which have been restricted by the 
obligate intracellular nature of Coxiella. However, the advent of a pure sample of the organism in question has been grown, using acidified citrate cysteine medium (ACCM), has transformed our capacity to examine this unique pathogen [11].

Query fever infection has been described in most countries except in New Zealand, Norway, Iceland and French Polynesia, report that they have not found any evidence of this organism in surveys to date. $Q$ fever is transmissible to man in whom it is characterized by sudden onset of fever, profuse sweating, chills, anorexia, malaise, myalgia, and sometimes nausea and vomiting etc. Most cases recover uneventfully but about 10 percent become chronic developing endocarditis and occasionally pericarditis. Cats, dogs, and wildlife may also serve as reservoirs hosts [12].

Coxiella burnetii has been identified in fish, rodents, wild birds, horses, marsupials, swine, marine mammals, camels, ducks, turkeys, geese, pets, and rabbits but was first isolated from a tick and has been identified in $\sim 40$ species of tick subsequently. The disease is subclinical in most animals, with adverse pregnancy events reportedly the most common clinical sign. Abortion rates can vary from a few cases up to $90 \%$ of the herd. In recent time $C$. burnetii has been identified as a biological threat agent due to its low infectious dose and high rate of transmission and is listed as a group B bioterrorism agent by the CDC in the US [13]. Few studies are conducted in Ethiopia indicated that 6.5\% seroprevalence of C. burnetii was observed in Addis Ababa abattoir workers [14]. And, seroprevalence of $31.6 \%, 90 \%$, and $54.2 \%$ of $C$. burnetii was recorded in cattle, camels and goats respectively in South Eastern Ethiopian pastoral zones of the Somali and Oromia regional states [15].

Even if most animals are remaining totally asymptomatic, including a lack of fever, low birth weight animals can occur [16]. Goats and sheep are the species in which abortions, stillbirths, and early neonatal mortality have been most frequently documented [17]. Doxycycline drug is the first line of treatment of $\mathrm{Q}$ fever for both animals and humans. Controlling environmental contamination through the control of infected ticks and biosecurity measures can reduce introduction of $C$. burnetii to the farms. Both antibiotic treatment and vaccination are the methods available for control and prevention of Coxiellosis. The objective of this seminar paper is to give highlight on various aspects of $Q$ fever in small ruminants with emphasis on its epidemiology, diagnosis, and its public health importance.

\section{Literature Reviews}

\section{Etiology}

The aetiological agent of $\mathrm{Q}$ fever is Coxiella burnetii, which is a Gram negative obligate intracellular bacterium, adapted to thrive within the phagolysosome of the phagocyte. It has been historically classified in the Rickettsiaceae family; however, recent phylogenetic investigations, based mainly on 16s rRNA sequence analysis, have shown that the Coxiella genus is distant from the Rickettsia genus in the alpha subdivision of Proteobacteria [18]. Coxiella burnetii has therefore been placed in the Coxiellaceae family in the order Legionellales of the gamma subdivision of
Proteobacteria. The complete genome sequencing of $C$. burnetii has been achieved and confirms its systematic position. Unlike rickettsiae family, Coxiella burnetii produces a small, dense, highly resistant spore-like form [19].

\section{Morphology, Characteristics, and History}

In August 1935, Davis and Cox, after E.H., Derrick had isolated in Queensland as the causative agent of Query fever, subsequently found the same organism as that found by Derrick. Soon after then, in 1939, the organism was named as Coxiella burnetii in honor of Cox and Burnet, who had identified the organism as a new Rickettsial [20]. It is an intracellular organism which is a polymorphic bacillus $(0.2-0.4 \mathrm{~mm}$ width, $0.4-1.0 \mathrm{~mm}$ length), which has a cell membrane, like Gram negative bacteria [21]. However, it stains poorly with pigmented Gram stain, but gimenez staining is traditionally used to stain the Coxiella burnetii pathogen from pathological materials and crop [22].

Coxiella burnetii has several distinctive characteristics, including a sporulation-like process that protects the organism against the external environment, where it can survive for long periods. In mammals, the usual host cell of $C$. burnetii is the macrophage, which is unable to kill the bacterium. The other an important characteristic of $C$. burnetii is its antigenic variations, those called phase variation. This antigenic shift can be measured and is valuable for differentiating acute from chronic $Q$ fever [23]. Thus, it displays two antigenic phases those are phase-I and phaseII that are liable to the Lipopolysaccharide (LPS) of the membrane. Phase I Coxiella burnetii antigens are corresponds to the smooth phase of Gram-negative bacteria and are more highly infectious and phase-II antigen is corresponding to the granular (Rough) phase which has a lower virulence. The strain of Coixella burnetii pathogens are grouped into six (I VI) genomic group based on the Restriction Fragment Length Polymorphism (RFLP). The pathogenicity and the virulence of the $C$. burnetii are associated with genetic characteristics, type of strains and plasmid groups [24] and with host factors such as pregnancy [25].

\section{Epidemiology}

Source, Maintenance and Transmission: Huge number of animal species are susceptible to infection by $C$. burnetii including ruminants, domestic carnivores, birds, wildlife mammals, and arthropods, such as ticks. Various species may play a role in the dissemination or maintenance of the disease, either as carriers or as vectors of $C$. burnetii, although the role of some of the species in the transmission of the disease has not been established with certainty. The source of human infections cannot frequently be established; however, sheep and goats are linked more frequently to Query fever outbreaks in humans than are other animal species [26].

Animal proximity and contact with infected animals and/ or their contaminated products (e.g. birth products) have been identified as important risk factors for humans. In most outbreaks, there are reports of spill-over of infection to humans from infected domestic small ruminants, i.e. goats $[27,28]$. The bacterial shed into the environment mainly occurs during parturition, during 
this period more than $10^{9}$ bacteria are released at the time of delivery. Goats mostly shed $C$. burnetii in vaginal mucus and milk, whereas sheep shed mostly in feces [29]. In goat's duration of shedding in milk is from several months to years. The udder and retro-mammary lymph nodes may remain infected for more than 20 months. Goats can become chronically infected and may shed for two consecutive $C$. burnetii pregnancies. Goats can shed $C$. burnetii in also placenta and vaginal mucus during two or more subsequent kidding. In contrast, ewes abort only one time, shed C. burnetii in vaginal mucus during the abortion, but do not shed in vaginal mucus at subsequent lambing [30]. The duration of excretion of $C$. burnetii may play a vital role in the persistence of infection. Human incident cases are considered the best reflector of disease activity globally, leading to intensified investigation into likely reservoirs.

Nevertheless, domestic small ruminants are considered the main source of human infection because they may shed $C$. burnetii in urine, feces, milk, and birth products. High concentrations of $C$. burnetii are found in the placenta and vaginal secretions of infected animals. Human infections occur after inhalation of aerosol or dust particles with parturient fluids of infected animals. In addition, bacterial survivability in adverse environmental condition and long-term persistence as pseudo spores are likely to give rise to the prevalence of enzootic-epizootic focus of Q fever [31].

\section{$\mathbf{Q}$ fever as a Biological Weapon}

Coxiella burnetii has been developed as a biological weapon. Query fever is a potential biological warfare agent being very infectious and very durable in the environment in a spore like form as well as capable of windborne spread. In addition to this, another route of transmission of $C$. burnetii could be through sabotage of the food supply. Many scientific advances were made proving that biological warfare was clearly feasible, although dependent on careful planning, especially about meteorological conditions. Large scale fermentation, concentration, purification, drying, stabilization, and weaponization of pathogenic microorganisms could be done safely.

In 1964, pathogens studied as biological weapons included the agents causing: - $Q$ fever, Anthrax, Glanders, Brucellosis, and a variety of animal pathogens and plant [32]. During the 1960s, bioweapon developers in the U.S. considered Q fever as an excellent "incapacitating" agent. The disease is debilitating, but rarely lethal. The U.S. military envisioned using $Q$ fever to cripple enemy forces and drain them of resources [33].

\section{Pathogenesis}

The pathogenesis of $C$. burnetii infection in humans and animals is not clearly understood. However, it is believed that bacterial LPS play an important role in the pathogenesis of $Q$ fever in both animals and humans [34]. The organism probably follows the oropharyngeal route as its port of entry into the lungs and intestine of both humans and animals [35]. The disease is highly infectious, and a very low dose is enough to initiate infection. Primary multiplication takes place in the regional lymph nodes after the initial entry, and a transient bacteraemia develops which persists for five to seven days, as shown in sheep [35].

The bacteria $C$. burnetii has two morphologically distinct cell variants: an intracellular, metabolically active Large Cell Variant (LCV) and a spore-like Small Cell Variant (SCV). These two forms are morphologically and functionally distinct. The LCV is larger, elongated less electron-dense bacteria and metabolically active and replicating by a large amount [36]. While the SCV presents a compact rod-shaped with a very dense central region, and it is considered as the metabolically dormant and less replicating. The SCVs are shed by infected animals. After infection the organism attaches to the cell membrane of phagocytic cells. After phagocytosis, the phagosome containing the SCV fuses with the lysosome [37].

The SCVs are metabolically activated in the acidic phagolysosome and can undergo vegetative growth to form LCVs [38,39]. The LCVs and the activated SVCs can be divided by binary fission and they can also undergo saprogenic differentiation. The spores that are produced can undergo further development to become metabolically inactive SCVs [40], and both spores and SCVs can then be released from the infected host cell by either cell lysis or exocytosis. The entire developmental cycle of metabolically active Coxiella burnetii takes place in acidic phagolysosome, $C$. burnetii is resistant to microbicidal activities in the host macrophages. The acidic environment also protects $C$. burnetii from the effects of antibiotics, as the efficacy of antibiotics is decreased in the acidic $\mathrm{pH}$. The SCV and spore forms are more difficult to denature than LCVs [41], possibly due to differences in cell wall composition and thickness as well as water content.

\section{Clinical Presentations}

In Small Ruminants: Coxiellosis in livestock species is generally asymptomatic. In animals, during the acute phase, C. burnetii can be found in the blood, spleen, lungs, and liver whereas during the chronic phase it is presented as a persistent shedding of $C$. burnetii in urine and feces. Most animals remain totally asymptomatic, including a lack of fever. However, low birth weight animals can occur. Goats and sheep are the species in which abortions, stillbirths, and early neonatal mortality have been most frequently documented. In many cases, abortion occurs in late pregnancy which range from 3 to $80 \%$ with unspecified characteristic clinical signs of infection with $C$. burnetii.

Temperature is an important factor related to abortion rates in herds, since fewer abortions take place between months of November and December. However, the occurrence of abortion rate increases gradually from January to February, decreasing again in March. The Aborted fetuses appear normal but, infected placentas exhibit intercotyledonary fibrous thickening and discolored exudates, which are not specific to Q fever [42]. A severe inflammatory response is noted in the myometrium of goats and goats shed $C$. burnetii in feces before and after kidding and the mean duration of excretion is 20 days. C. burnetii can also be recovered from milk for up to 32 months. 
In Humans: Coxiella burnetii infection can cause either an asymptomatic, acute, or chronic disease. Size of the inoculums, route, and duration of exposure, as well as host factors influence the duration of the incubation period and may contribute to the clinical expression of acute and chronic clinical disease. Monocytes and macrophages are the primary target cell [43]. Inhalation of aerosolized bacteria through the respiratory tract is the Primary route of infection, with alveolar macrophages being targeted. Kupffer cells of the liver are affected through bacteremia or, rarely, digestive route exposure. Only $4 \%$ of those affected with acute $\mathrm{Q}$ fever need hospitalization for their symptoms. The incubation period prior to acute clinical $\mathrm{Q}$ fever is estimated at approximately 20 days (range: 12 to 39 days) [44] depending on inoculating dose [45].

The mean incubation period between the onset of clinical signs and acute $Q$ fever diagnosis is estimated at 2 months. Acute $\mathrm{Q}$ fever is seen as a self-limiting mild febrile illness and may be associated with headache, myalgia, arthralgia, and coughing [46]. Prolonged fever is usually accompanied by severe headaches may reach from 39 to $40.8{ }^{\circ} \mathrm{C}$, usually remaining elevated all day. Fever typically increases to plateaus within 2-4 days and returns to normality within 5-14 days in treated individuals. Untreated patients may observe fever of 5-57 days duration. Atypical pneumonia is one of the most commonly recognized forms of acute $\mathrm{Q}$ fever. Most cases are clinically asymptomatic or mild, characterized by a nonproductive cough, fever, and minimal auscultatory abnormalities, but some patients present with acute respiratory distress [47].

Hepatitis is also commonly observed, which is usually accompanied clinically by fever and less frequently by abdominal pain, anorexia, vomiting, nausea, and diarrhea. Advanced icterus and palpation of a mass in the right hypochondrium have also been reported. Rarely, dermatological and neurologic manifestations occur [48]. The annual mortality rates in the general population associated with acute clinical Q fever range from 0.9 per 100,000 per year in the United Kingdom to 2.4 per million per year in France (Figure 1). Myocarditis is the leading cause of death in acute disease and accounts for $2 \%$ of all clinical cases [49].

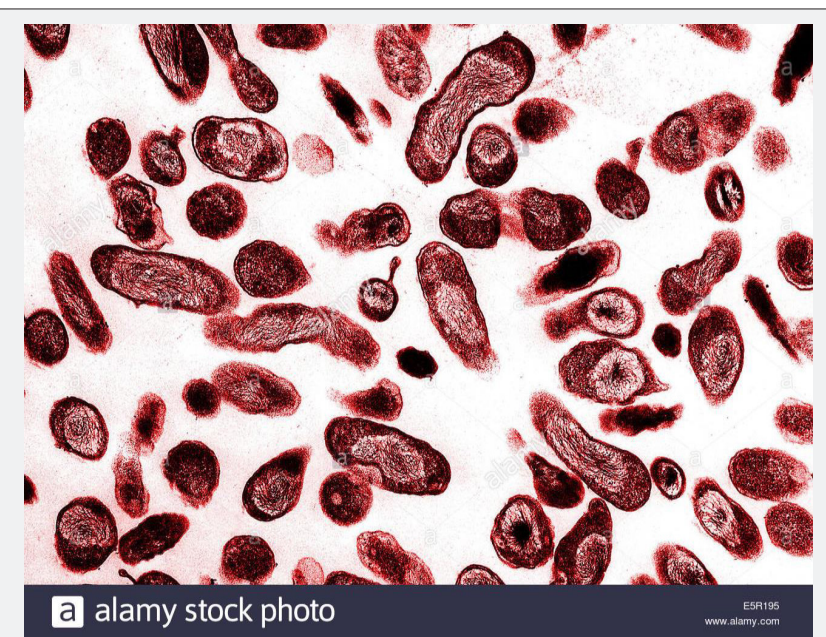

Figure 1: Electron microscope image of coxiella burnetti.

Acute disease does not have to precede chronic $\mathrm{Q}$ fever. Five percent (5\%) of chronic $Q$ fever occurs in infected individuals [50] and is characterized by persistent infection beyond six months. It happens almost exclusively in high risk patients, including those with immunosuppression, vascular abnormalities, heart valve lesions, and pregnancy. The prognosis of $Q$ fever is very poor, if it is not treated [51]. Clinical indications of prolonged persistence of $Q$ fever (chronic Q fever) include; osteo-articular infections, chronic hepatitis, infection of the ventriculo-peritoneal drain [52], pseudo tumors of the spleen and lung [53], and chronic fatigue syndrome [54]. In general sense, the clinical signs in human are the ones listed below: Rash, Pregnancy (week by week, trimesters), Headache, Diarrhea, Abdominal Pain, Pneumonia, Influenza like syndrome, Meningoencephalitis, Hepatitis, Endocarditis, etc.

\section{Diagnosis}

Collection of Samples: In the acute-phase of the disease, immediately serum sample should be collected after the onset of symptoms (within the first 2 weeks) with a convalescentphase specimen collected 3-6 weeks later. Before antibiotic administration the whole blood should be collected in EDTAtreated anticoagulant tubes and shipped refrigerated on the frozen gel packs by overnight. The Buffy coat can be saved for DNA amplification and stored frozen in a non-frost-free freezer if samples are to be prepared for other laboratory tests. The most commonly evaluated sample used for confirmation for chronic Q fever is heart valve tissue. If the fresh samples being transported within 24 hours they should be refrigerated and shipped on frozen gel packs. However, if the transport does not occur within 24 hours, it should be frozen in a non-frost-free freezer and shipped on dry ice for either culture or PCR analysis [55].

Staining and Related Measures: The diagnosis of coxiellosis in aborted small ruminants by an ordinary method is to detect the pathogen using staining techniques. The smears prepared from the samples are usually stained by Gimenez, Stamp, Giemsa stain or Machiavello. Strong presumptive diagnosis of $C$. burnetii is indicated by the presence of large masses of red-colored coccobacilli. Due to possible confusion with other pathogens such as Brucella species or Chlamydia species, these diagnostic 
methods are poorly sensitive and not specific for Q fever [56]. This is followed by a serological analysis by the CFT, or better by ELISA [57]. However, staining techniques cannot be specific and they have reduced sensitivity, especially with vaginal swabs, milk and a fecal sample.

Isolation and Cultivation: For routine diagnosis of $\mathrm{Q}$ fever cultivation of $C$. burnetii is not recommended, since the process is very difficult, time consuming, and dangerous. In addition to this culture of $C$. burnetii requires a bio-safety level 3 (BSL3) laboratory because bacteria are highly infective and can be hazardous for laboratory workers. Patients with chronic $Q$ fever have already received antibiotics which can further complicate isolation attempts; a negative culture does not rule out a $C$. burnetii infection. Specimens can be referred to CDC through state public health laboratories for culture.

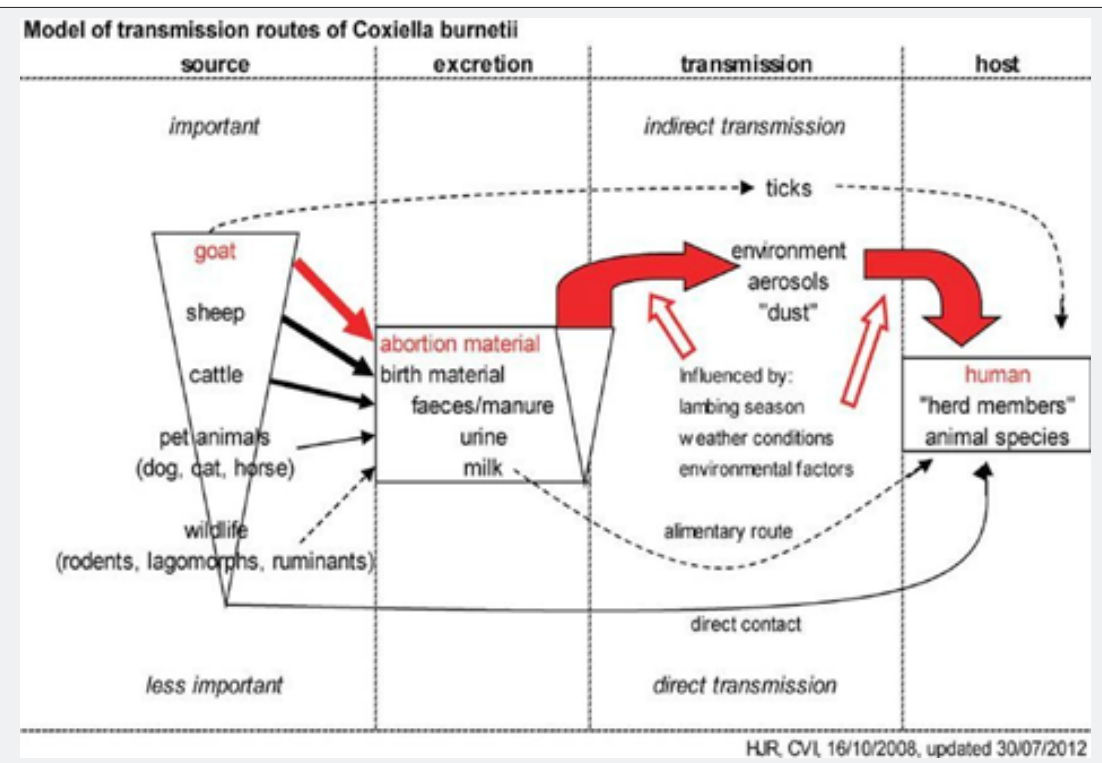

Figure 2: Model of transmission routes of Coxiella burnetiid.

Serology: The commonest serological tests presently used for the diagnosis of $C$. burnetii in animals are the compliment fixation test (CFT), immunofluorescence assay (IFA) and enzyme linked immunosorbent assays (ELISA) [58]. The CFT has lower sensitivity as a diagnostic test for Q-fever compared to IFA and ELISA tests which are highly specific and sensitive to both phase I and phase II antigens [58]. Both IFA and ELISA tests were used in the present study and compared as herd screening tests. The IFA assays employ fluorescent markers conjugated to a specific antibody to detect antigen-antibody (Ag: $\mathrm{Ab}$ ) reaction. In positive cases, the fluorescent marker in the Ag: Ab complex emits a green light which is detected under the fluorescent microscope. The two available tests for serological diagnosis of $\mathrm{Q}$ fever are direct and indirect types of test. The latter is the one more commonly used. On the other hand, the most commonly used ELISA assay for screening $C$. burnetii infection in ruminants is an indirect test which utilizes a horseradish peroxidase-labeled monoclonal antiruminant IgG conjugate that reacts with a wide range of domestic and wild ruminant species [59] (Figure 2).

Molecular Method: For study transmission routes, and to assess sources of infection of $C$. burnetii, molecular typing of pathogenic microorganisms is mainly important. Use of antibiotics and vaccination may interfere with the structure of bacterial populations and this can be evaluated by comparing molecular typing profiles of members of these populations. The prerequisite for surveillance purposes and epidemiological investigation of $\mathrm{Q}$ fever outbreaks is genotypic characterization of $C$. burnetiid [60]. Two PCR C. burnetii typing methods have been described. Those are 1, Multi-Locus Variable number of tandem repeats Analysis (MLVA) and 2, Multispacer Sequence Typing (MST) that permit the typing of $C$. burnetii without the need for isolation of the organism [61].

Multispacer Sequence Typing, depending on DNA sequence variations in10 short intergenic regions, can be performed on isolated C. burnetii strains or directly on extracted DNA from clinical samples. To date, the most discriminating methods for C. burnetii those allowing the identification of up to 36 distinct genotypes are MLVA and MST. The availability of such databases allows inter-laboratory comparisons to be made easily and this will lead to a better understanding of the propagation of the $C$. burnetii isolates. Furthermore, their use in the characterization of field samples or isolates is increasing [62].

\section{Differential Diagnosis}

The disorders for which we appreciate the same signs with that of Q fever are: Elective Abortion, Influenza, and Rickettsial Infection. At initial stages, i.e., before pulmonary symptoms are present, influenza may be suspected. Furthermore, Salmonellosis, Brucellosis, Leptospirosis, Campylobacteriosis, and less severe disease due to Rickettsial species should be included in differential diagnosis. Malaria and Dengue should have to be excluded, especially in tropical areas [63].

\section{Public Health Importance}

Query fever is essentially an airborne disease which is caused by $C$. burnetii. Human infections occur after inhalation of aerosols 
generated from infected placentas, body fluids or contaminated dust resulting from contaminated manure and desiccation of infected placenta. Transmission of $C$. burnetii is mostly associated with abortion of domestic small ruminants, and particularly with ovine abortions. Seasonal variation in the incidence of the human disease in the spring and early summer IS described by a several authors which has been attributed to spring lambing and shearing which in turn leads to environmental contamination [64].

Indeed, people may be infected by handling contaminated wool, manure or clothes contaminated with feces or transhumance of infected flocks through a valley [65]. Then direct contact with aborted females is not required. In addition, between June and November 2002, in the Chamonix valley in France, 88 human cases of Q fever did identify, 71 with clinical signs and 10 need hospitalizations. The origin of this outbreak due to airborne transmission from some infected flocks present in the valley since then. In the USA, C. burnetii is enzootic in wild animals and ruminants as in other parts of the world, but human infections due to $C$. burnetii are rare [66]. Certain cases of $Q$ fever have been described in Australia [67], Canada [68], France, Germany [69], Japan [70], Spain, Switzerland and the United Kingdom [71].

Except in Bulgaria where the rise of the number of goats that daily go through villages and small towns for grazing is associated with an increase in human $\mathrm{Q}$ fever, a few information is available to explain such an increase of cases of $Q$ fever [72]. A recent study has also confirmed that, $\mathrm{Q}$ fever has a significant long-term health impact with more than one in to 24 months post diagnosis. Since the multiple forms of the disease hinder its clinical diagnosis, the prevalence of $Q$ fever in humans as well as in animals is not known accurately and is probably underestimated. The existence of $C$. burnetii in the environment allows it to be disseminated by wind far away from its original source. This indicate the appearance of $\mathrm{Q}$ fever cases in urban areas, where an important percentage of patients fails to report direct contact with animals [73].

Apparently without animal contact, domestic and wild birds can also be responsible for human cases in urban areas since they are able to transmit $\mathrm{Q}$ fever via their feces or their ectoparasites. The airborne transmission of $C$. burnetii associated with its highly resistance to environments and the ability to easily produce huge quantities of $C$. burnetii in the after birth of aborted ewes or goats have led to classify $C$. burnetii as a category-B, biological terrorism agent [74]. Then it was perhaps used for this purpose during World War II. The classification C. burnetii under biological terrorism agent has been responsible for the publication of several reviews on $\mathrm{Q}$ fever.

The attention of public health personnel and medical on $Q$ fever, which could be responsible for the apparent increase of $Q$ fever cases and its apparent reemergence, has been focused. Less efficient route of contamination is ingestion of contaminated raw milk or raw milk products. Without clinical signs, seroconversion in human volunteers has been induced by drinking of contaminated milk, but none of them presented aggravating risk factors. Nevertheless, some studies have reported clinical disease linked to the ingestion of cheese; but these outcomes are sometimes contested since it is difficult to guarantee even for prisoners that the patients did not inhale contaminated dust or aerosols [75].

\section{Treatments}

Treatment is indicated for all infections, even for those that are subclinical. For domestic small ruminants' oral therapeutic dose may be given for 24 weeks [76]. Doxycycline is the first choice of drugs treatment for all adults, and children those have severe illness. Treatment should be initiated immediately whenever $\mathrm{Q}$ fever is suspected. The other use of antibiotics other than doxycycline for 14 to 21 days at a dosage of $100 \mathrm{mg}$ twice daily to adults and $2.2 \mathrm{mg} / \mathrm{kg}$ body weight twice daily to children under $45 \mathrm{~kg}$ or other tetracycline, at a dose $8 \mathrm{mg} / \mathrm{kg}$ in drinking water, several weeks before lambing, was recommended as a possible prevention protocol is also associated with a higher risk of severe illness [77].

For preventing the development of severe complications, doxycycline is most effective if it is treatment started earlier in the course of disease. In some cases, such as, survival in endovascular complications such as mycotic aneurysm or vascular graft infections surgical intervention may be necessary. Surgical debridement is also prescribed for osteo-articular infections. After the fever subsides patients should be treated for at least 3 days until there is evidence of clinical improvement. As stated by CDC [78], the standard duration of treatment is 2-3 weeks [79]. Children who is less than 8 years of age with mild illness should be treated with cotrimoxazole, but therapy should be switched to doxycycline if their course of illness worsens. Trimoxazole treatment of pregnant women diagnosed with acute $\mathrm{Q}$ fever with once daily throughout pregnancy significantly decreases the risk of adverse consequences for the fetus. In adults $100 \mathrm{mg}$ of doxycycline in every 12 hours and $200 \mathrm{mg}$ of hydroxychloroquine in every 8 hours is indicated for Chronic $Q$ fever. Standard duration of treatment is 18 months [80].

\section{Prevention and Control}

The best methods available for prevention and control of Coxiellosis are antibiotic treatment and vaccination. In-feed addition of tetracycline or injectable oxytetracycline pre-partum has not been shown to prevent $C$. burnetii shedding in feces, milk and vaginal secretions [81]. Due to the lack of enough evidence that supporting antibiotic efficacy and the fact that prudent use of antibiotics is needed to avoid resistance, they should not be used for treatment of $\mathrm{Q}$ fever in animals at this time.

Vaccination with a phase-I C. burnetii, inactivated vaccine is reportedly effective for abortion prevention, and the reduction of bacterial shedding in goats and sheep [82] and is most effective in primiparous animals. If animal is already infected with $C$. burnetii vaccination may not be effective [83]. In Australia, phase-I vaccine is currently available for human use and has been recommended for high risk, occupationally exposed, sero negative individuals. Great attention should be given to $C$. burnetii since it can survive for several weeks outside a host in warm, moist environments. 
Bearing in mind small ruminants as the main reservoirs, controlling endemic Coxiellosis in herd may play a crucial role in reducing disease in populations living in close contact with infected animals (Table 1). Controlling environmental contamination Table 1: Shows $Q$ fever status in Ethiopia.

\begin{tabular}{|c|c|c|c|c|}
\hline Years & Place of Study & Study Group & Notes \\
\hline 1990 & Addis Ababa & Abattoir workers & 6.5 & $6.5 \%$ of abattoirs workers in Addis Ababa Abele A. (1990) \\
\hline $2008-2010$ & South-east Ethiopia & Various Animal species & $31.6-90$ & $31.6 \%$ of cattle, 90\% of camels and 54.2\% of goats in Southeast \\
Ethiopia Gumi et al. (2013)
\end{tabular}

During transport of manure it should be covered, stored in a location where water run-off and water contamination is minimal. To reduce the risk of spread through aerosolization, transport and spread of manure should be done when wind is minimal [85]. Composting carcasses and aborted tissues and contaminated bedding on site is preferred [86]. Vaccination offers a new conception of suppression and eradication of this Zoonosis, not only in a view of public health safety but also in creating $Q$ fever free regions in endemic areas [87].

\section{Status of Q Fever in Ethiopia}

As a few studies conducted in Ethiopia indicated that $6.5 \%$ seroprevalence of $C$. burnetii was observed in Addis Ababa abattoir workers. Also, the existence of antibody against $C$. burnetii was reported in goats and sheep slaughtered at Addis Ababa abattoir, and its peri-urban zones [88]. A seroprevalence of $31.6 \%, 90 \%$, and $54.2 \%$ of $C$. burnetii was recorded in cattle, camels and goats respectively in South Eastern Ethiopian pastoral zones of the Somali and Oromia regional states. Ticks were tested for $C$. burnetii in Ethiopia by quantitative real time polymerase chain reaction targeting two different genes followed by multispacer sequence typing (MST). An overall prevalence of $6.4 \%$ of $C$. burnetii was recorded. C. burnetii was detected in $28.6 \%$ of Amblyomma gemma, 25\% of Rhipicephalus pulchellus, $7.1 \%$ of Hyalomma marginatum rufipes, $3.2 \%$ of Amblyomma variegatum, $3.1 \%$ of Amblyomma cohaerens, $1.6 \%$ of Rhihipicephalus praetextatus, and $0.6 \%$ of Rhipicephalus (Boophilus) decoloratus. Significantly higher overall frequencies of C. burnetii DNA were observed in Amblyomma gemma and Rhihipicephalus pulchellus than in other tick species [89].

\section{Conclusion and Recommendations}

Coxiellosis (Query fever) is a zoonotic disease caused by an obligate intracellular bacterium known as $C$. burnetii. This disorder has repeatedly been described since the 1930s and cannot be defined yet as an emerging zoonotic disease. Re-emergence of this case could be explained by the improvement of the diagnosis for which serological tests and PCR allow an accurate detection of the infected flocks $[90,91]$. The high prevalence of acute $Q$ fever could reflect such vigilance even if the mandatory notification through the control of infected ticks and biosecurity measures may reduce introduction of $C$. burnetii to the farms. The preferred method for handling manure and bedding and decreases the Coxiella burden and risk of spread is composting [84]. of $\mathrm{Q}$ fever by physicians, veterinarians and laboratories does not exist wherever it has been undertaken. This tells us that mandatory notification is not always the accurate solution for a better knowledge of its epidemiology. Nowadays, this measure is not desirable in veterinary medicine until more data on the disease become available in order to propose validated methods to efficiently prevent the transmission of the disease to humans. In order to precisely identify the origin of each human infection and to better understand the mechanisms paving a way to various clinical manifestations in humans and small ruminants, in fact, it is necessary to obtain more data on the bacteria, on molecular markers and virulence factors.

As it has been tried to be mentioned in the above review, most of the outbreaks of $Q$ fever in humans are related to goats and sheep, but until now it has not been identified whether or not, the $C$. burnetii isolated from sheep are more virulent than those isolated from goats and any others, or why sheep preferentially shed $C$. burnetii in vaginal mucus and feces, whatever the virulence of the strain could be. Furthermore, the efficiency of antibiotic treatments in small ruminants on bacterial shedding must be well evaluated. To conclude, it is advisable to inform the persons at risk: farmers, veterinarians, livestock workers, immunodeficient patients or those suffered from cardiac valvopathy and pregnant women that they must avoid contact with ewes and goats during delivering their lambs and kids, even cows during delivery period.

\section{Recommendations}

Based on the above conclusion the following measures should be taken into account for prevention; control and management of $\mathrm{Q}$ fever as far as all above characters are concerned:

a. Lambing and kidding areas should be regularly cleaned and disinfected to prevent accumulation of potentially contaminated materials and it is preferable that birthing should occur indoors away from the wind.

b. Be sure that the placenta and fetus from aborted goats and sheep are submitted for examination to an accredited veterinary diagnostic laboratory in order to test for $C$. burnetii. Because, placenta is the key sample to submit since the organism may not be detectable in the fetus. 
c. All personal hygienic measures should be taken into consideration and every livestock owner should manage his own herd in well applicable manner.

d. Pregnant women and highly at-risk person should not assist, even should avoid contact in lambing or kidding; infants and young children, the elderly and immunocompromised persons should be given care.

e. Mammals, in effect, domestic small ruminants should be immunized through vaccination which is effective for controlling infection and transmission since there is no practical means for eradication of $C$. burnetii from the environment.

f. Further research should be conducted regarding Q fever in small ruminants in Ethiopia so that it may help in designing the mitigating approaches.

\section{References}

1. Maurin M, Raoult D (1999) Q fever. Clin Microbiol Rev 12: 518-553.

2. Bielawska Drózd A, Cieślik P, Mirski T, Bartoszcze M, Knap JP (2013) Q fever-selected issues, Annals of Agricultural and Environmental Medicine 20: 222-232.

3. Cutler SJ, Bouzid M, Cutler RR (2007) Q fever J Infect 54(4): 313-318.

4. Van Asseldonk MAPM, Bontje DM, Backer JA, van Roermund HJW, Bergevoet RHM (2015) Economic aspects of Q fever control in dairy goats. Preventive Veterinary Medicine 121: 115-122.

5. Arricau Bouvery N, Souriau A, Lechopier P, Rodolakis A (2003) Experimental Coxiella burnetii infection in pregnant goats: Excretion routes. Vet Res 34: 423-433.

6. Van Loenhout JA, Hautvast JL, Vercoulen JH, Akkermans RP, Wijkmans CJ, et al. (2015) Q fever patients suffer from impaired health status long after the acute phase of the illness: from a 24-month cohort study. J Infec 70(3): 237-246.

7. Delsing CE, Warris A, Bleeker Rovers CP (2011) Q fever: still more queries than answers. In Hot Topics in Infection and Immunity in Children VIII. Springer New York, USA, pp. 133-143.

8. Vander Hoek W, Morroy G, Renders NH, Wever PC, Hermans MH, et al. (2012) Epidemic Q fever in humans in the Netherlands. In C. burnetii: Rec Adv New Persp Res Q fevers Bacter. Netherlands, pp. 329-364.

9. Van Asseldonk MAPM, Prins J, Bergevoet RHM (2013) Economic assessment of $Q$ fever in the Netherlands. Prev Vet Med 112(1): 27-34.

10. Madariaga MG, Rezai K, Trenholme GM, Weinstein RA (2003) Q fever: a biological weapon in your backyard. Lanc Infect Dis 3(11): 709-721.

11. Omsland A, Cockrell DC, Howe D, Fischer ER, Heinzen RA (2009) Host cell free growth of the $\mathrm{Q}$ fever bacterium Coxiella burnetii. Proc Nat Ac Sci 106(11): 4430-4434.

12. Greenslade E, Beasley R, Jennings L, Woodward A, Weinstein P (2003) Has Coxiella burnetii ( $Q$ fever) been introduced into NZ? Emerg Infect Dis 9(1): 138-140.

13. Oyston PCF, Davies C (2011) Q fever: the neglected biothreat agent. J med Microbiol 60(1): 9-21.

14. Abebe A (1990) Prevalence of $Q$ fever infection in the Addis Ababa abattoir. Ethio Med J 28 (3): 119-122.

15. Gumi B, Rebuma Firdessa, Lawrence Yamuah, Teshale Sori, Tadele Tolosa, et al. (2013) Seroprevalence of Q fever in Southeast Ethiopian Pastoral Livestock. J Vet Sci Med Diagn 2: 1.
16. Marrie TJ, Stein A, Janigan D, Raoult D (1996) Route of infection determines the clinical manifestations of acute $Q$ fever J Infect Dis 173: 484-487.

17. Porter SR, Czaplicki G, Mainil J, Guattéo R, Saegerman C (2011) Q fever: current state of knowledge and perspectives of research of a neglected Zoonosis. Int J Microbial.

18. Drancourt M, Raoult D (2005) Genus I. Coxiella. In: Bergey's Manual of Systematic Bacteriology, East Lansing, MI, USA, 2: 237-241.

19. McCaul TF, Williams JC (1981) Developmental cycle of Coxiella burnetii: structure and morphogenesis of vegetative and sporogenic differentiations. J Bacteriol 147: 1063-1076.

20. Davis R (2004) Microsoft power point presentation on $Q$ fever. Funded by Center for Disease Control (CDC). Iowa State University, Ames, Iowa.

21. Kovacova E, Kazar J (2002) Q fever still a query and underestimated infectious disease. Acta Virol 46: 193-210.

22. Hollenbeck BL, Gannon S, Qian Q Grad Y (2015) Genome sequence and analysis of resistance and virulence determinants in a strain of Neisseria mucosa causing native-valve endocarditis. JMM Case Reports, 2: 3 .

23. Matthieu Million, Didier Raoult (2013) Hunter's Tropical Medicine and Emerging Infectious Disease. pp. 558-560.

24. Eldin C, Mahamat A, Demar M, Abboud P, Djossou F, Raoult D (2014) Q fever in French Guiana. Am J Trop Med Hyg 91(4): 771-776.

25. Toman R, Heinzen RA, Samuel JE, Mege JL (2012) Coxiella burnetii: Recent advances and new perspectives in research of the $Q$ fever bacterium pp. 984.

26. McQuiston JH, Childs JE (2002) Q fever in humans and animals in the United States. Vector borne and zoonotic diseases, 2(3): 179-191.

27. Schimmer B, TerSchegget R, Wegdam M, Zuchner L, de Bruin A, et al. (2010) The use of a geographic information system to identify a dairy goat farm as the most likely source of an urban Q fever outbreak. BMC Infect Dis 10(1): 69.

28. Hellenbrand W, Schoenberg I, Pfaff G, Kramer M, Breuer T (2005) The relevance of Coxiella burnetii infections in animals for $Q$ fevers in humans-measures for prevention and control. Tierarz Prax Großt 33(1): 5-11.

29. Rodolakis A, Berri M, Hechard C, Caudron C, Souriau A, et al. (2007) Comparison of $C$. burnetii shedding in milk of dairy bovine, caprine, and ovine herds. J Dair Sci 90(12): 5352-5360.

30. Berri M, Laroucau K,Rodolakis A (2000) The detection of Coxiella burnetii from ovine genital swabs, milk and fecal samples by the use of a single touchdown polymerase chain reaction. Vet Microbial 72 (3): 285-293.

31. Lyytikainen O, Ziese T, Schwartlander B, Matzdorff P, Kuhnhen C, et al. (1998) An outbreak of sheep associated $Q$ fever in a rural community in Germany. Eu J Epidemiol 14(2): 193-199.

32. SIPRI (Stockholm International Peace Research Institute) (1971) The Rise of CB Weapons. In: The Problems of Chemical and Biological Warfare. Humanities Press, New York, USA.

33. CCA (Council of Canadian Academies) (2012) Healthy Animals, Health Canada. The expert panel on approaches to animal health risk assessment. Cribb A, Dohoo IR, Donahue D, Fairbrother JM, (Eds.), Council of Canadian Academies, Ottawa, Canada.

34. Angelakis E, Raoult D (2010) Q fever. Vet Microbiol 140(3): 297-309.

35. Woldehiwet Z (2004) Q fever (coxiellosis): epidemiology and pathogenesis. Res Vet Sci 77(2): 93-100.

36. Bennett MD, Bahazis MJ (2014) Chapter I: C. burnetii. Manual of Security Sensitive Microbes and Toxins. Liu D (Eds.), CRC. Press, USA, pp. 333-350. 
37. Williams JC, Thompson HA (1991) Q fever: The Biology of Coxiella burnetii. CRC Press, Boca Raton, FL, ISBN-13: 9780849359835. pp. 368.

38. Roest HIJ (2013) Coxiella burnetii in pregnant goats. Ph.D. Thesis, Department of Bacteriology and TSEs, C. Vet Inst Wageningen, UR, Lelyst Neth pp. 229

39. Roest HI, Bossers A, van Zijderveld FG, Rebel JM (2013) Clinical microbiology of Coxiella burnetii and relevant aspects for the diagnosis and control of the zoonotic disease. Q fever Vet Q, 33(3):148-160.

40. Van Schaik EJ, Chen C, Mertens K, Weber MM, Samuel JE (2013) Molecular pathogenesis of the obligate intracellular bacterium Coxiella burnetii. Nature Rev Microbial 11(8): 561-573.

41. Scott GH, Williams JC (1990) Susceptibility of Coxiella burnetii to chemical disinfectants. Annls. New York, USA, Ac Sci 590(1): 291-296.

42. Arricau Bouvery N, Rodolakis A (2005) Is Q fever an emerging or reemerging zoonosis Vet. Res. 36(3):327-349.

43. La Scola B, Lepidi H, Raoult D (1997) Pathologic changes during acute $Q$ fever: influence of the route of infection and inoculum size in infected guinea pigs. Infec Immu 65(6): 2443-2447.

44. Lopez E, Ascher M, Roberto R, Chin J (1986) Q fever among slaughterhouse workers: California. MMWR, 35(14): 223.

45. Marrie TJ, Raoult D (1997) Q fever a review and issues for the next century. Int J Antimic Agents, 8(3): 145-161.

46. Tissot Dupont H, Raoult D (2007) Studies on Q fever in man. Tran Assoc Am Phys 69: 98-104.

47. Raoult D, Levy PY, Harle JR, Etienne J, Massip P, et al. (1990) Chronic Q fever. Diagnosis and follow up. Ann. N.Y Acad Sci 590: 51-60.

48. Bernit E, Pouget J, Janbon F, Dutronc H, Brouqui P (2002) Neurological involvement in acute $Q$ fever: a report of 29 cases and review of the literature. Arch Int med 162(6): 693-700.

49. Fournier PE, Etienne J, Harle JR, Habib G, Raoult D (2001) Myocarditis, a rare but severe manifestation of $Q$ fever: report of 8 cases and review of the literature. Clin Infect Dis 32(10): 1440-1447.

50. Fenollar F, Fournier PE, Raoult D (2004) Molecular detection of Coxiella burnetii in the sera of patients with endocarditis or vascular infection. J Clin Microbiol 42(11): 4919-4924.

51. Botelho Nevers E, Fournier PE, Richet H, Lepidi H, Raoult D (2007) Coxiella burnetii infection of aortic aneurysms or vascular grafts: report of 30 new cases and evaluation of outcome. European J Clin Microbiol and Infect Dis 26(9): 635-640.

52. Lohuis PJ, Ligtenberg PC, Diepersloot RJ, De Graaf M (1994) Q-fever in a patient with a ventriculo-peritoneal drain. Case report and short review of the literature. The Neth J med 44(2): 60-64.

53. Lipton JH, Fong TC, Gill MJ, Burgess K, Elliot PD (1987) Q fever inflammatory pseudo tumor of the lung. Chest 92: 756-757.

54. Raoult D, Tissot-Dupont H, Foucault C, Gouvernet J, Fournier PE, et al. (2000) Q fever 1985-1998: clinical and epidemiologic features of 1,383 infections. Med 79(2): 109-123.

55. Hamilton LR, George DL, Scoville SL, Hospenthal DR, Griffith ME (2011) PCR for rapid diagnosis of acute $Q$ fever at a combat support hospital in Iraq. Milit med 176(1): 103-105.

56. Berri M, Rekiki A, Boumedine KS, Rodolakis A (2009) Simultaneous differential Detection of Chlamydophila abortus, Chlamydophila pecorum and Coxiella burnetii from aborted ruminant clinical samples using multiplex PCR. BMC microbiol. 9(1):130.

57. Kovacova E, Kazar J, Spanelova D (1998) Suitability of various C. burnetii antigen preparations for detection of serum antibodies by various tests. Actavirol.42 (6): 365-368.

58. Slaba K, Skultety L, Toman R (2005) Efficiency of various serological techniques for diagnosing Coxiella burnetii infection. Acta Virol. 49(2): 123-127.

59. Hussein MF, Al-Khalifa IM, Aljumaah RS, Gar Elnabi A, Macasero WV (2012) Serological prevalence of Coxiella burnetii in captive wild ruminants in Saudi Arabia. Comparative Clinical Pathology, 21(1): 3338.

60. Glazunova O, Roux V, Freylikman O, Sekeyova Z, Fournous G (2005) C. burnetii genotyping. Emerg Infect Dis 11: 1211-1217.

61. Svraka S, Toman R, Skultety L, Slaba K, Homan WL (2006) Establishment of a genotyping scheme for Coxiella burnetii. FEMS, microbial. Lett 254(2): 268-274.

62. Chmielewski T, Sidi Boumedine K, Duquesne V, Thierry R, Tylewska Wierzbanowska (2009) Molecular epidemiology of Q fever in Poland. Polish Journal of microbiology, 58(1): 9-13.

63. Krauss H, Schieffer HG, Slenczka W (2003) Zoonosis: infectious diseases transmissible from animals to humans. Am Soc Microbiology.

64. Tissot Dupont H, Torres S, Nezri M, Raoult D (1999) Hyperendemic focus of Q fever related to sheep and wind. Am J Epidemiol 150(1): 6774.

65. Dupuis G, Petite J, Peter O, Vouilloz M (1987) An important outbreak of human Q fever in a Swiss Alpine valley Int J Epidemiol 16(2): 282-287.

66. Gami AS, Vera S Antonios, Rodney L Thompson, Hari P Chaliki, Naser M Ammash (2003) Q fever Endocarditis in the United States. Mayoclinicproceedings.org. Volume 79(2): 253-257.

67. Yohannes K, Roche P, Gidding H, Bunn C, Della Porta T (2004) Australia's notifiable diseases status: Annual report of the National Notifiable Diseases Surveillance System. Communic Dis Intell Quart Rep 28(1): 6

68. Langley JM, Marrie TJ, LeBlanc JC, Almudevar A, Resch L, et al. (2003) Coxiella burnetii seropositivity in parturient women is associated with adverse pregnancy outcomes. Am J Obst Gynecol 189(1): 228-232.

69. Hellenbrand W, Breuer T, Petersen L (2001) Changing epidemiology of Q fever in Germany 1947-1999. Emerg Infect Dis 7(5): 789.

70. Takahashi H, Tokue Y, Kikuchi T (2004) Prevalence of community acquired respiratory tract infections associated with Q fever in Japan, Diagn. Microbiol Infect Dis 48: 247-252.

71. Pebody RG, Wall PG, Ryan MJ, Fairley C (1996) Epidemiological features of $C$. burnetii infection in England and Wales: 1984 to 1994. Communic Dis rep CDR rev 6(9): 128-132.

72. Lyytikainen O, Ziese T, Schwartlander B, Matzdorff P, Kuhnhen C, et al. (1997) Outbreak of Q fever in Lohra Rollshausen, Germany. Euro surveillance: Eu Commun Dis Bullet 2(2): 9-11.

73. Sampere M, Font B, Font J, Sanfeliu I, Segura F (2003) Q fever in adults: review of 66 clinical cases. Eu J Clin Microbiol Infect Dis 22(2): 108110 .

74. Kagawa FT, Wehner JH, Mahindra V (2003) September. Q fever as a biological weapon. In Sem in resp infect 18(3): 183-195.

75. Fishbein DB, Raoult, D (1992) A cluster of Coxiella burnetii infections associated with exposure to vaccinated goats and their unpasteurized dairy products. Am J Tropi Med Hyg 47(1): 35-40.

76. Kahn CH (2005) The Merck Veterinary Manual, ( $9^{\text {th }}$ edn), Merck and Co INC. WHS, USA.

77. Blain S (2007) Contagious diseases of ruminant management of Q fever in goats. Summa, Animali da Reddito, 2(3): 59-63.

78. Hartzell JD, Wood Morris RN, Martinez LJ, Trotta RF (2008) Q fever: epidemiology, diagnosis, and treatment. In Mayo Clin Proc 83(5): 574 579.

79. CDC (Center for Disease Control and prevention (2016) Bioterrorism and the pediatric patient: an update. Clinical Pediatric Emergency Medicine, 14(2): 102-117. 
80. Carcopino X, Raoult D, Bretelle F, Boubli L, Stein A (2009) Q fever during Pregnancy. Ann. NY Aca Sci 1166(1): 79-89.

81. Astobiza I, Jesús F Barandika, Hurtado Ramón A. Juste, García Pérez (2010) Kinetics of Coxiella burnetii excretion in a commercial dairy sheep flock after treatment with oxytetracycline. The Veterinary Journal, 184(2): 172-175.

82. Guatteo R, Seegers H, Taurel AF, Joly A, Beaudeau F (2011) Prevalence of Coxiella burnetii infection in domestic ruminants: a critical review. Vet Microbiol 149(1): 1-16.

83. Rousset E, Durand B, Champion JL, Prigent M, Dufour P, et al. (2009) Efficiency of a phase 1 vaccine for the reduction of vaginal Coxiella burnetii shedding in a clinically affected goat herd. Clin Microbiol Infect 15(2): 188-189.

84. Schelling E, Diguimbaye C, Daoud S, Nicolet J, Boerlin P, et al. (2003) Brucellosis and Q-fever seroprevalences of nomadic pastoralists and their livestock in Chad. Prev Vet Med 61(4): 279-293.

85. Tissot Dupont H, Amadei MA, Nezri M, Raoult D (2004) SWind in November, Q fever in December. Emerg Infect Dis 10(7): 1264.
86. Menzies PI, Falzon LC, Shakya KP, Jones Bitton A, Peregrine AS (2013) Anthelmintic resistance in sheep flocks in Ontario, Canada. Vet Parasitol 193(1):150-162.

87. EFSA (2010) Panel on Animal Health and Welfare (AHAW). Scientific opinion on Q fever. Eu FS Auth J 8(5): 1595.

88. Philip CB, Hoogstraal H, Reiss-Gutfreund R, Clifford CM (1966) Evidence of rickettsial disease agents in ticks from Ethiopian cattle. Bull World Health Organ 35: 127-131.

89. Kumsa B, Socolovschi C, Almeras L, Raoult D, Parola P (2015) Occurrence and Genotyping of Coxiella burnetii in Ixodid Ticks in Oromia, Ethiopia. Am J Trop Med Hyg 93(5): 1074-1081.

90. Stephen Berger books (2018) edition. Infectious Diseases of Ethiopia: Q fever pp. 242 of 345.

91. Tissot Dupont H, Raoult D, Brouqui P, Janbon F, Poirier R (1992) Epidemiologic features and clinical presentation of acute $Q$ fever in hospitalized patients: 323 French cases. The Am J med 93(4): 427-434

Your next submission with Juniper Publishers
will reach you the below assets
- Quality Editorial service
- Swift Peer Review
- Reprints availability
- E-prints Service
- Manuscript Podcast for convenient understanding
- Global attainment for your research
- Manuscript accessibility in different formats
( Pdf, E-pub, Full Text, Audio)
- Unceasing customer service
Track the below URL for one-step submission
https://juniperpublishers.com/online-submission.php

